\title{
Evaluation of Official Healthcare Informatics Applications in Saudi Arabia and their Role in Addressing COVID-19 Pandemic
}

\author{
Nouf Alassaf ${ }^{1}$, Sulaiman Bah $^{2}$, Fatima Almulhim ${ }^{3}$, Norah AlDossary ${ }^{3}$, Munirah Alqahtani ${ }^{4}$ \\ 'Department of Health Information Management \& Technology, College of Applied Medical Sciences, Imam Abdulrahman Bin Faisal University, Jubail, Saudi Arabia \\ ${ }^{2}$ Department of Public Health, College of Public Health, Imam Abdulrahman Bin Faisal University, Dammam, Saudi Arabia \\ ${ }^{3}$ Cancer Registry Unit, Oncology Centre, King Fahad Specialist Hospital, Dammam, Saudi Arabia \\ ${ }^{4}$ Tumor Registry Unit, Oncology Institute, Johns Hopkins Aramco Healthcare, Dhahran, Saudi Arabia
}

Objectives: The purpose of this study was to examine official healthcare informatics applications in Saudi Arabia in the context of their role in addressing the coronavirus disease 2019 (COVID-19) pandemic. Methods: This is a case study of official healthcare informatics programs and applications (apps) developed in Saudi Arabia before and during the COVID-19 pandemic. The qualitative content analysis (QCA) method was used. Data collection consisted of two components: a desktop review of documents and actual testing of the programs. According to the QCA method, we developed a matrix for abstracting information on different apps and programs in order to categorize the data. The compilation of information and discussion were based on information summarized in the matrix. Results: Six apps in total were developed before the COVID-19 pandemic. With the advent of the COVID-19 pandemic, three of the apps, SEHA, Mawid, and Sehaty were modified to address different aspects of the pandemic. Both SEHA and Mawid included information about COVID-19 awareness. During the COVID-19 pandemic, three official apps were developed: Tawakkalna, Tetamman, and Tabaud. The Tawakkalna app is mandatory for all citizens and residents to activate when visiting stores and institutions. It has a wide range of COVID-19 and other health-related functions. The Tetamman app provides COVID-19 test results and allows one to check his or her daily symptoms. It also has an educational content library and provides alerts. The Tabaud app notifies individuals if they have been exposed to COVID-19. The features, advantages, and disadvantages of all of the apps were examined. Conclusions: Overall, there were more strengths than shortcomings in the role played by healthcare informatics in the handling of the COVID-19 pandemic in Saudi Arabia.

Keywords: Informatics, Delivery of Health Care, COVID-19, Public Health, Saudi Arabia

Submitted: August 25, 2020

Revised: 1st, November 24, 2020; 2nd, March 4, 2021

Accepted: April 23, 2021

\section{Corresponding Author}

Sulaiman Bah

Department of Public Health, College of Public Health, Imam Abdulrahman Bin Faisal University, Dammam 34212, Saudi Arabia. Tel: +966-562812189, E-mail: sbah@iau.edu.sa (https://orcid.org/00000002-7621-211X)

This is an Open Access article distributed under the terms of the Creative Commons Attribution Non-Commercial License (http://creativecommons.org/licenses/by$\mathrm{nc} / 4.0 /$ ) which permits unrestricted non-commercial use, distribution, and reproduction in any medium, provided the original work is properly cited.

(c) 2021 The Korean Society of Medical Informatics

\section{Introduction}

The unprecedented speed at which coronavirus disease 2019 (COVID-19) was declared a pandemic warranted concerted efforts for dealing with the disease from multiple fronts, including health informatics. COVID-19-related apps were developed from all parts of the globe, coming from both middle-income and developing countries. Silva categorized COVID-19-related apps into five clusters: informational, self-assessment/medical reporting, contact tracing, multipurpose apps, and others [1]. In the Arabian Gulf countries of Oman, Kuwait, Bahrain, and United Arab Emirates, a total 
of six COVID-19-related apps were developed from March 2020 to April 2020. All these apps used global positioning system (GPS) and Bluetooth technology to enhance preventive measures through the inclusion of a feature for tracing positive cases and notifying people nearby of potential exposure to the virus [1]. As with other countries in the Arabian Gulf, Saudi Arabia also took several healthcare initiatives to address COVID-19 [2]. While extensive research has been conducted on the COVID-19 pandemic, there have been no studies of the healthcare informatics sector in the context of the pandemic. The purpose of this paper is to fill this gap in research and, more specifically, to examine the official healthcare informatics applications implemented in Saudi Arabia in light of their role in addressing the COVID-19 pandemic.

\section{Methods}

This is a case study of official healthcare informatics programs and apps developed in Saudi Arabia before and during the COVID-19 pandemic. The inductive approach of qualitative content analysis (QCA) was the method used [3]. The data used come from a desktop review of documents and actual testing of the programs. Information was retrieved from the Ministry of Health $(\mathrm{MOH})$ website, via published literature, and via the gray literature (such as news items, internal memos, official announcements, etc., in English and Arabic). The Google search terms used to identify other COVID-19-related apps developed elsewhere included "COVID-19 apps" and "Corona apps."

According to the QCA method, analysis of the data obtained on various software programs was carried out through categorization and compilation [3]. For categorization, we developed a matrix for abstracting information about the different apps and programs.

The compilation of data and discussion were based on information summarized using this matrix. For benchmarking, we reviewed COVID-19-related apps developed elsewhere, paying special attention to apps developed in neighboring Arabian Gulf countries due to their similar socio-economic and cultural contexts.

\section{Results}

The details of the official apps developed by the government before COVID-19 pandemic are shown in Table 1, and the apps developed to address the COVID-19 pandemic after it had already begun are shown in Table 2. Six apps had been developed before the COVID-19 pandemic: the Health Electronic Surveillance Network (HESN) (2012), 937 (2013), SEHA (2017), Asafny (2017), Mawid (2017), and Sehhaty (2019). The apps were all developed by the MOH, except for Asafny, which was developed by Red Crescent. The $\mathrm{MOH}$ apps are targeted to all citizens and residents, while the Red Crescent app is a stand-alone app that is targeted only to those seeking emergency care via the Red Crescent. HESN is a special electronic system for public health and disease surveillance. As of 2021, over 47 communicable disease groups are exclusively reported using HESN. The 937 app provides 24/7 telephone-based health services for both medical emergencies and regular health consultations, while the SEHA app is designed to offer online medical consultation services [4]. The Asafny app is used to provide a quick response to people in emergency situations, and the Mawid app is used to book and manage medical appointments. Lastly, the Sehaty app links patients to relevant health information and provides them with medical consultations with a variety of health professionals.

With the advent of the COVID-19 pandemic, in addition to the older $\mathrm{MOH}$ apps being modified to address the pandemic, new apps were developed as well. HESN was modified in April 2020 to allow for easy reporting of COVID-19 cases and characteristics related to the case. It is mandatory by law in Saudi Arabia for healthcare centers to report all cases of communicable diseases, including COVID-19, through the HESN system. Both SEHA and Mawid were modified to provide information about COVID-19 awareness. SEHA provides assessment services that employ artificial intelligence methods, while Mawid has a self-assessment survey. In addition, Mawid includes options for accessing 937 or SEHA. Sehaty also allows users to book a COVID-19 test appointment if they have an account with Mawid.

During the COVID-19, three new official apps were developed in 2020: Tetamman (March), Tawakkalna (April), and Tabaud (June). It is mandatory for all citizens and residents to activate the Tawakkalna app whenever visiting stores and institutions during the pandemic. It has many services, including tools for requesting travel permits when curfews are in place, requesting personal permits, requesting gathering permits, checking the status of a COVID-19 test, accessing education services, and accessing dependent services. The Tetamman app provides COVID-19 test results and allows one to check for symptoms on a daily basis. It also has an educational content library and provides alerts. The Tabaud app notifies individuals if they had been exposed to someone with a confirmed COVID-19 case. It also enables individuals 


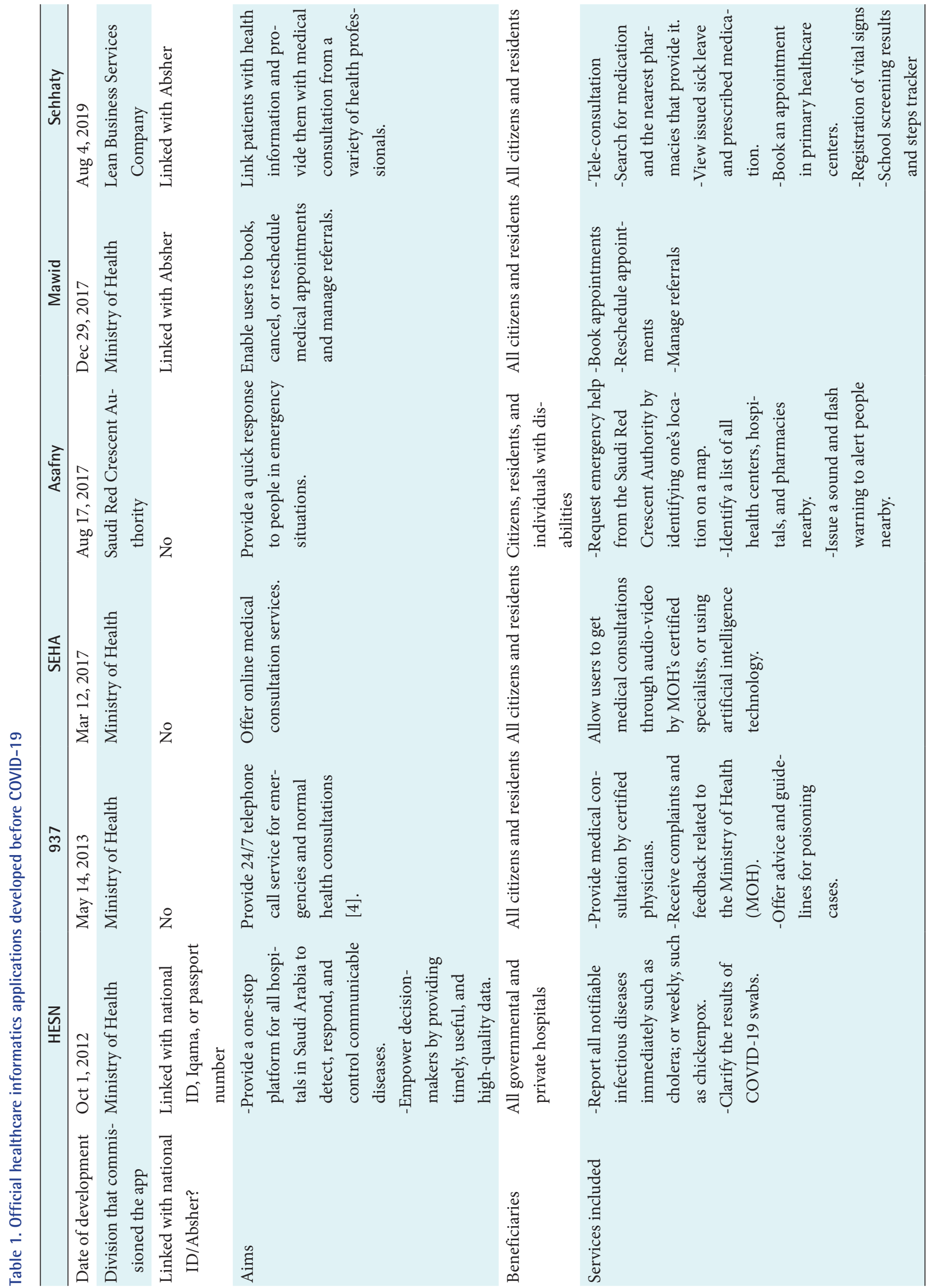




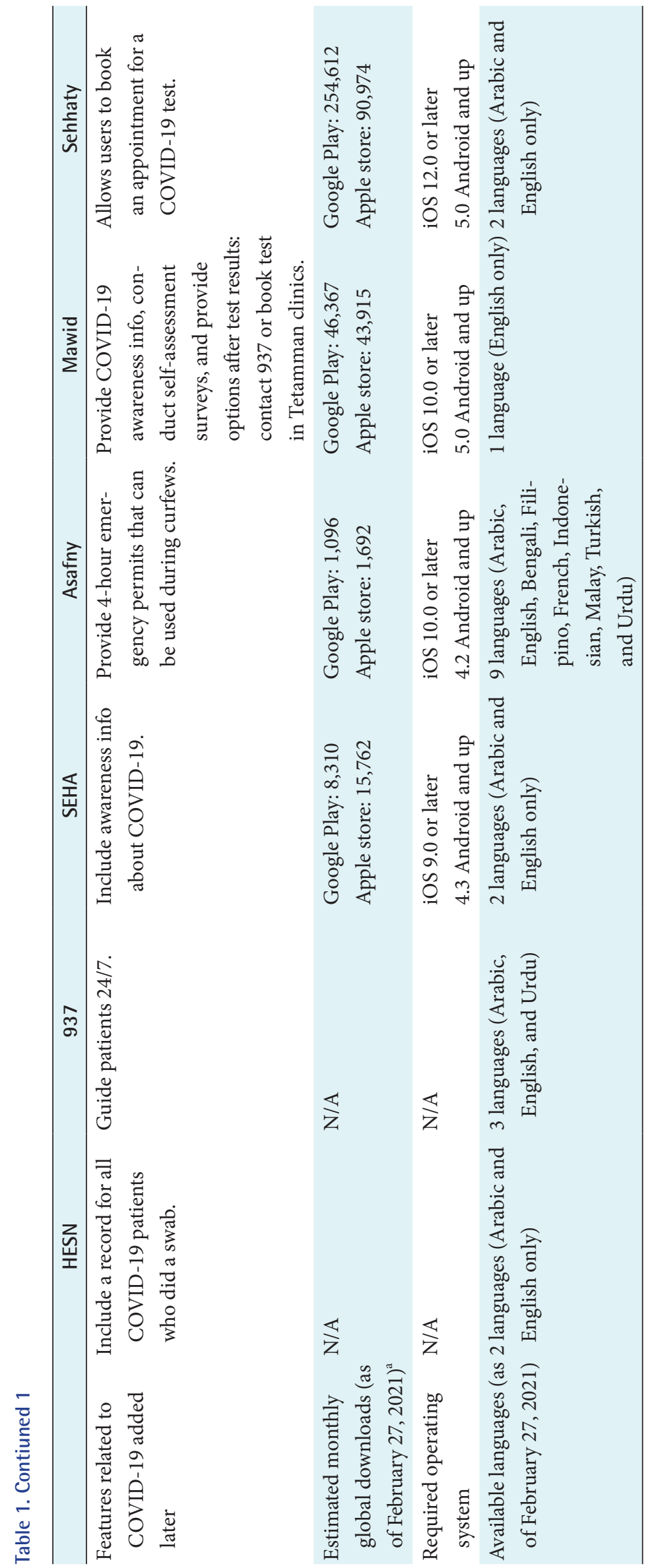




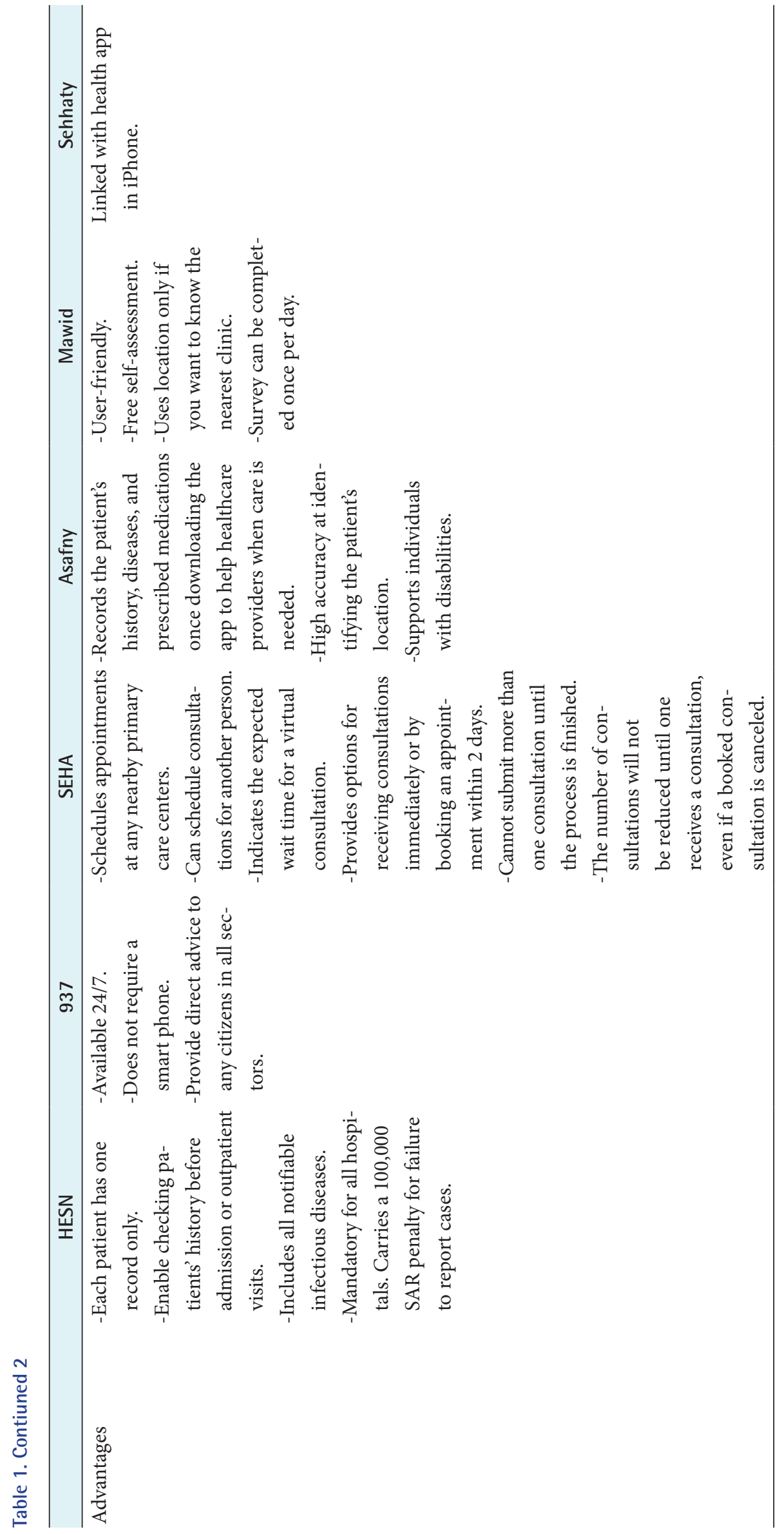




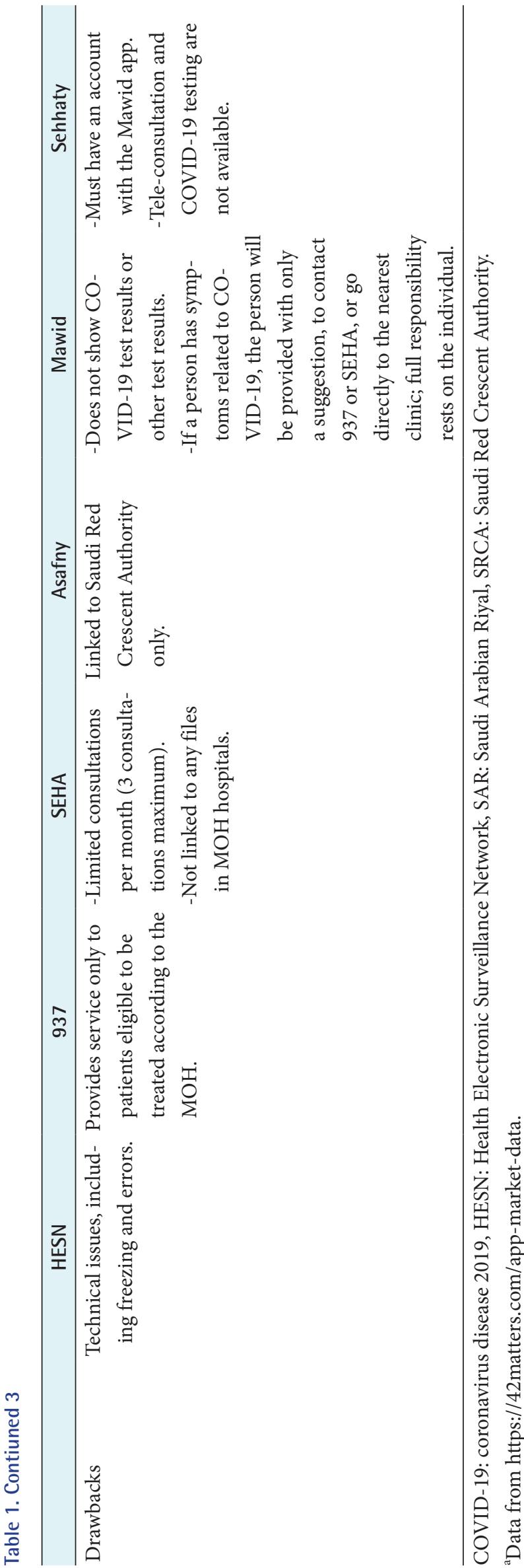

to voluntarily share their results among people with whom they had contact in the previous 14 days. The Tetamman app was developed by the $\mathrm{MOH}$ while the Tabaud app was developed by the Saudi Data and Artificial Intelligence Authority (SDAIA).

\section{Discussion}

During the COVID-19 pandemic, Saudi Arabia used several healthcare informatics apps among the multiple tools to mitigate the effects of the pandemic. Some of the apps were developed specifically to address different aspects of the COVID-19 pandemic, while other were developed before the pandemic. Our examination of the role of healthcare informatics in Saudi Arabia's COVID-19 response uncovered both positive and negative points. The adaptation of HESN for reporting COVID-19 cases is a positive decision as it helps to standardize data collection for rapid analysis. Similarly, the adaptation of older apps designed before the COVID-19 pandemic to address certain aspects of the pandemic was also a positive decision as existing resources were able to be used. The development of three apps during the COVID-19 pandemic was very successful. The Tawakkalna app was broader and highly inclusive, while the Tatamman and Tabaud apps had more specific functions. Tabaud is comparable to other similar apps developed in the Gulf region, such as BeAware (Bahrain) and Shlonik (Kuwait). Unlike these apps, Tabaud, relies on Bluetooth technology rather than GPS technology, which is an advantage of the Saudi Arabia-developed COVID-19 apps. The emergence of the SDAIA as a developer of and stakeholder in Saudi Arabian healthcare informatics is a merit. This similar to the way that COVID-19-related apps were developed in Bahrain, where the BeAware app was developed by the Information and eGovernment Authority, and in Kuwait, where the Shlonik app was developed by the Kuwait Central Agency for Information Technology.

A few shortcomings were observed as well. The HESN was only adapted to address factors related to COVID-19 in April 2020. Prior to this, data related to COVID-19 from the HESN system were unstructured and disorganized. Tabaud was launched in June 2020, 2 months after the launch of the Gulf Cooperation Council apps. There is a technical issue with the Tawakkalna app that results in a delay of several days before a user infected with COVID-19 is shown as being infected in the app using the color-coded system. This delay can have a potentially damaging effect, as the infected person might expose others to the virus during this time 


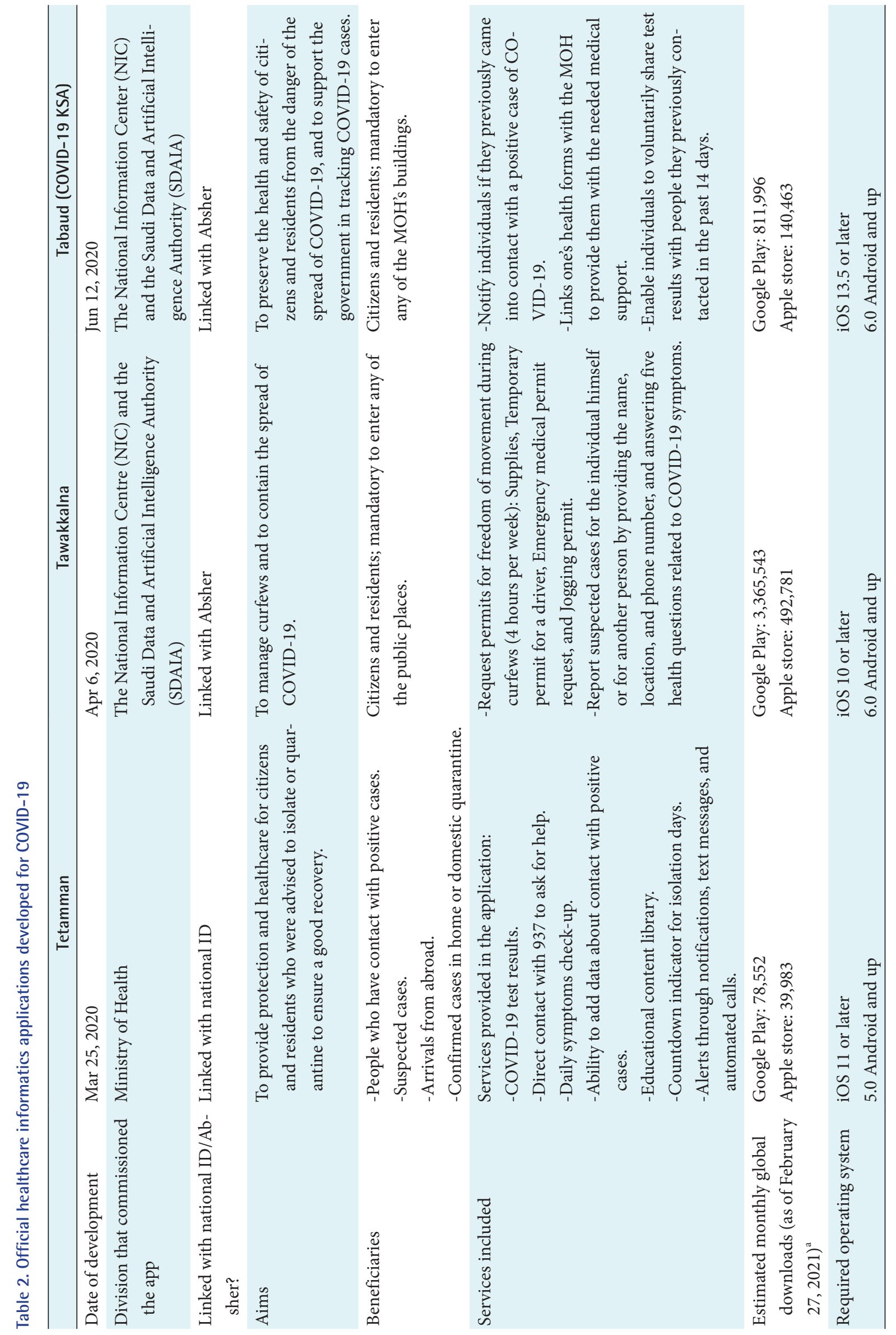




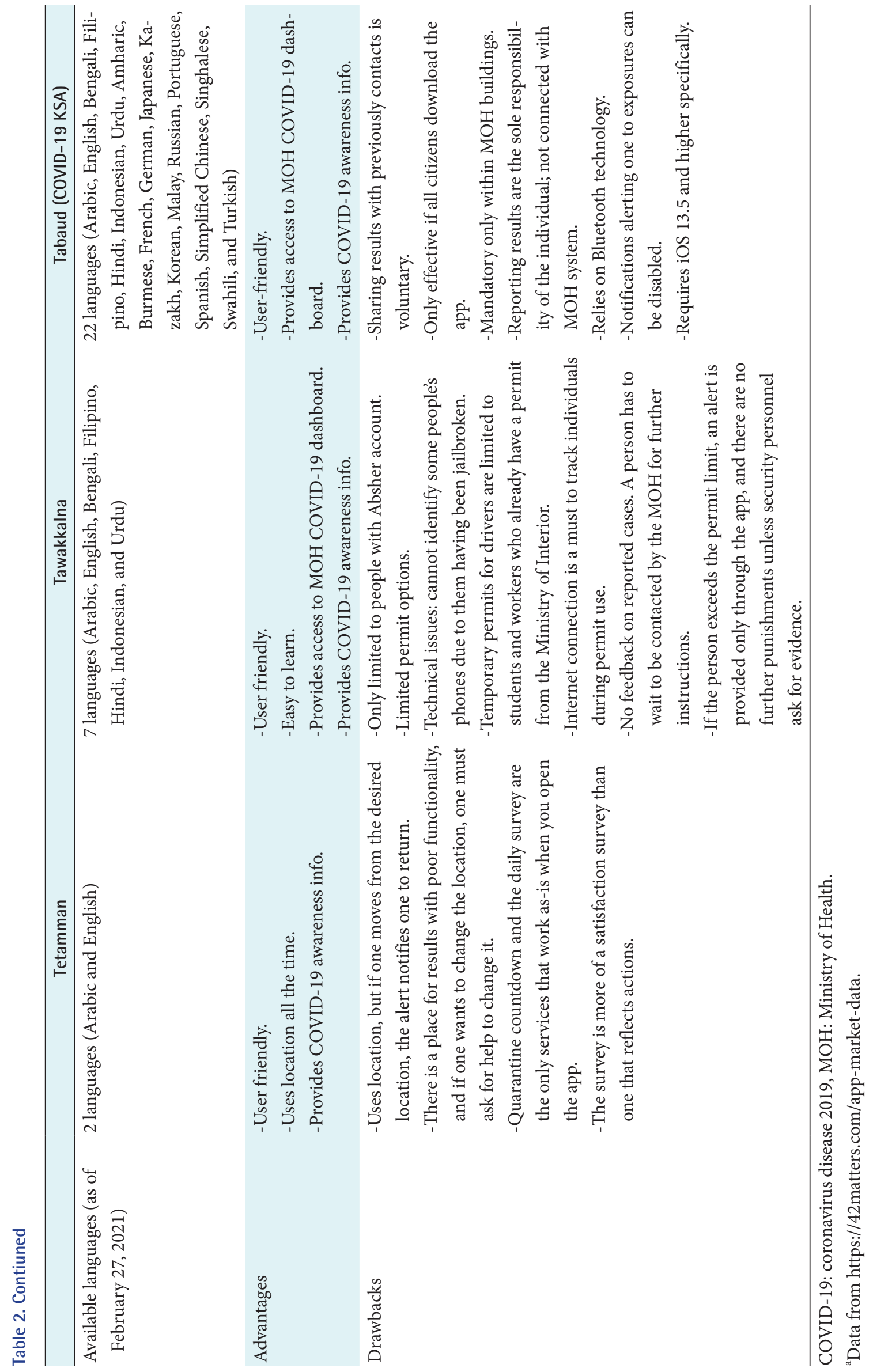


without being officially deemed "infected." Lastly, while some of the apps include interconnected features, one integrated app would be more effective than several loosely connected apps. In Qatar, Bahrain, Kuwait, and Oman only one app was used for COVID-19 surveillance, assessment, and awareness.

In conclusion, the purpose of this paper was to examine the official healthcare informatics in Saudi Arabia in the context of their role in addressing the COVID-19 pandemic. Overall, we found that there were more strengths than shortcomings in the role played by healthcare informatics in Saudi Arabia's handling of COVID-19. While six apps were developed from 2012 to 2019, three apps were developed within a span of only 3 months during the COVID-19 pandemic, and all of the different apps served different needs. With the emergence of COVID-19, old apps were adapted to address factors related to the pandemic. While most of the apps developed during the pre-COVID-19 era were commissioned by the Ministry of Health, a new stakeholder, the SDAIA, has entered the field with the emergence of COVID-19. We see this as a positive development that is similar to the approach adopted by other Arabian Gulf countries. One study limitations is the frequency with which apps are updated, which may result in differences between the present-day status of apps and the status of apps as described in this study and the lack of centrality for accessing information about the apps discussed in this study.

\section{Conflict of Interest}

No potential conflict of interest relevant to this article was reported.

\section{ORCID}

Nouf Alassaf (https://orcid.org/0000-0002-3962-4457)

Sulaiman Bah (https://orcid.org/0000-0002-7621-211X)

Fatima Almulhim (https://orcid.org/0000-0002-0247-5371)

Norah AlDossary (https://orcid.org/0000-0002-6111-8200)

Munirah Alqahtani (https://orcid.org/0000-0003-1119-2354)

\section{References}

1. Silva MA. Covid-19 apps (EENA 112) [Internet]. Brussels, Belgium: European Emergency Number Association; 2020 [cited at 2021 Jun 29]. Available from: https:// eena.org/document/covid-19-apps/.

2. Global Health Exhibition. Saudi Arabia's digital healthcare landscape post-COVID-19 [Internet]. Riyadh, Kingdom of Saudi Arabia; Global Health Exhibition; c2021 [cited at 2021 Jun 29]. Available from: https:// www.globalhealthsaudi.com/en/overview/saudi-news/ Saudi-Arabias-digital-healthcare-landscape-post-COVID-19.html.

3. Bengtsson M. How to plan and perform a qualitative study using content analysis. NursingPlus Open 2016;2:8-14.

4. Ministry of Health. 937 services [Internet]. Riyadh, Kingdom of Saudi Arabia: Ministry of Health; 2021 [cited at 2021 Jun 29]. Available from: https://www.moh. gov.sa/en/937/Pages/default.aspx. 